\title{
COMPARAÇÃO DE DUAS METODOLOGIAS MULTIVARIADAS NO ESTUDO DE SIMILARIDADE ENTRE FRAGMENTOS DE FLORESTA ATLÂNTICA ${ }^{1}$
}

\author{
Rinaldo Luiz Caraciolo Ferreira², Adalmeres Cavalcanti da Mota ${ }^{3}$, José Antônio Aleixo da Silva², Luiz \\ Carlos Marangon ${ }^{2}$ e Eufrázio de Souza Santos ${ }^{5}$
}

\begin{abstract}
RESUMO - O objetivo deste trabalho foi comparar duas metodologias multivariadas no estudo de similaridade entre fragmentos de Mata Atlântica. Foi realizado um levantamento bibliográfico, e a partir de 11 fragmentos de Floresta Atlântica, localizados nos Estados de Pernambuco, do Rio Grande do Norte, de Minas Gerais, de São Paulo e do Rio de Janeiro, montaram-se os bancos de dados para a realização do estudo da similaridade florística empregando duas metodologias de técnicas de análise multivariada. Na metodologia usual foi utilizada uma matriz binária (presença/ausência) de 236 espécies arbóreas ocorrentes nos 11 fragmentos, bem como se realizou uma análise de agrupamento, utilizando o método da ligação simples e o coeficiente de Jaccard. Na metodologia proposta foi empregada a análise de componentes principais para redução da dimensão da matriz de densidades e dominâncias absolutas das 236 espécies arbóreas, utilizando-se os escores desses componentes principais para aplicar a análise de agrupamento, por meio do método de ligação simples e da distância euclidiana. Foram identificados dois agrupamentos: um com fragmentos da Região Nordeste (Pernambuco) e outro com fragmentos da Região Sudeste (Minas Gerais). $\mathrm{Na}$ metodologia proposta foi identificado apenas um grupo com fragmentos da região nordestina (Pernambuco), ressaltando-se que as variáveis quantitativas são de suma importância para a associação das matas em diferentes regiões. A metodologia proposta apresentou potencial para utilização no estudo de similaridade de fragmentos florestais.
\end{abstract}

Palavras-chave: Análise de agrupamento, análise de componentes principais e associação de técnicas multivariadas.

\section{COMPARISON OF TWO MULTIVARIATE METHODOLOGIES IN SIMILARITY STUDIES AMONG ATLANTIC FOREST FRAGMENTS}

\begin{abstract}
The objective of this work was to compare two multivariate methodologies in similarity studies among Atlantic Forest fragments. A bibliographical survey was carried out, and databanks were set up from 11 fragments of Atlantic Forest located in the States of Pernambuco, Rio Grande do Norte, Minas Gerais, São Paulo and Rio de Janeiro. The study of the floristic similarity was constructed using two methodologies of the multivariate analysis. For the usual methodology, a binary matrix (presencelabsence) of 236 tree species present in the 11 fragments was used. A cluster analysis was applied using the simple linkage method and the Jaccard's coefficient. In the proposed methodology, the principal components analysis was used for reducing the size of the density matrix and absolute dominancy of the 236 tree species, using the principal component scores to apply the cluster analysis by the Euclidean distance single linkage method. Two groups were identified; one in the Northeastern Region (Pernambuco) and another in the Southeastern Region (Minas Gerais). The proposed methodology identified only one group with fragments in the Northeastern region (Pernambuco), standing out that the quantitative variables are of utmost importance for the association of forests in different regions. The proposal methodology has potential for use in the study of forest fragment similarity.
\end{abstract}

Keywords: Cluster analysis, principal components analysis and association of multivariate techniques.

\footnotetext{
${ }^{1}$ Recebido em 04.06.2007 e aceito para publicação em 19.05.2008.

${ }^{2}$ Departamento de Ciência Florestal da Universidade Federal Rural de Pernambuco (UFRPE), Recife-PE. E-mail:<rinaldo@ dcfl.ufrpe.br>.

${ }^{3}$ Departamento de Estatística e Informática da UFRPE. E-mail: <eufrazio@ufrpe.br>.
} 


\section{INTRODUÇÃO}

A delimitação dos domínios da Mata Atlântica sempre gerou muita discussão entre pesquisadores. Segundo Joly et al. (1991), desde as primeiras divisões fitogeográficas propostas para o Brasil, diversos autores consideraram seus limites de maneiras muito diferentes, utilizando diferentes caracteres florísticos, fisionômicos, estruturais, (fito)geográficos e (fito)climáticos. Evidenciou-se a necessidade de padronização de técnicas de análise de similaridade que sirvam para detectar relações entre as comunidades e os ambientes, através dos agrupamentos formados segundo as variáveis analisadas (VAN TONGEREN, 1987).

A falta de padronização metodológica entre estudos de florística e fitossociologia realizados no Brasil, bem como em outros lugares do mundo, é apontada por muitos autores como uma dificuldade para a realização de comparações seguras que contribuiriam muito para um melhor entendimento dos padrões observados (FERREIRA e PRANCE, 1998; DURINGAN et al. 2000; DISLICH et al. 2001; SANTOS, 2003).

A utilização de métodos multivariados para comparar a similaridade entre formações florestais vem sendo adotada, porém estudos com esses métodos ainda são poucos em número e regionais no escopo, ou tratam apenas de uma formação vegetal (OLIVEIRA-FILHO e RATTER, 1995; VANDER BERG O OLIVEIRA-FILHO, 2000; OLIVEIRA-FILHO EFONTES, 2000; SCUDELLER et al. 2001, PEIXOTO et al., 2004)

Segundo Scudeller (2002), um dos primeiros estudos utilizando análises multivariadas para comparar a similaridade florística de localidades que incluía áreas da Mata Atlântica foi o de Silva e Shepherd (1986), que realizaram uma análise de agrupamento considerando os gêneros presentes em 32 localidades e comentaram que as diversas classificações e denominações da Mata Atlântica ao longo de sua distribuição geográfica indicam que existem diferenças marcantes na composição florística entre diferentes trechos.

A análise de agrupamento com base em levantamentos florísticos vem sendo a técnica mais usual na comparação de similaridade entre formações florestais baseada na presença e ausência de espécies (SCIAMARELLI, 2005). No entanto, embora levantamentos estritamente florísticos, quando considerados em grande número, sejam muito úteis para uma análise inicial da vegetação de determinada área e permitam comparações amplas baseadas na presença e ausência de espécies, é necessário incluir medidas de abundância, quando se pretendem detalhar as comparações, principalmente, entre áreas com floras semelhantes (SCUDELLER, 2002).

O objetivo deste presente trabalho foi comparar duas metodologias de comparação de similaridade entre fragmentos de Mata Atlântica.

\section{MATERIAL E MÉTODOS}

\subsection{Dados}

Primeiramente, foi realizado um levantamento bibliográfico de estudos quantitativos alocados em área do bioma Mata Atlântica. Os trabalhos encontrados neste levantamento passaram por uma seleção, visando a alguma padronização dos dados a serem extraídos. Os critérios utilizados para a seleção dos trabalhos foram os seguintes: a) indicação da formação vegetacional levantada; b) realização do levantamento em uma área limitada e indicação da localização e tamanho da área levantada; c) especificação do método de levantamento usado e critério de inclusão de indivíduos arbóreos com diâmetro a 1,30 m do solo (DAP) $\geq 5$ $\mathrm{cm}$ ou circunferência a $1,30 \mathrm{~m}$ do solo $(\mathrm{CAP}) \geq 15,8$ $\mathrm{cm}$; d) identificação de pelo menos $50 \%$ do material coletado até o nível de espécies; e) levantamentos que dispusessem dos parâmetros fitossociológicos: densidades absoluta (DA) e relativa (DR), frequiências absoluta (FA) e relativa (FR), dominâncias absoluta (DoA) e relativa (DoR) e valor de importância (VI); e f) levantamentos realizados entre os anos de 2000 e 2006. Com base nesses critérios, selecionaram 11 referências bibliográficas (Quadro 1).

\subsection{Preparação e Análise dos Dados}

Com a seleção dos trabalhos foi confeccionada uma lista de 237 espécies que apresentaram oito ou mais indivíduos, considerando-se que as espécies menos abundantes influenciam muito pouco os padrões resultantes de análises multivariadas de dados quantitativos de vegetação e aumentam sem necessidade o volume dos cálculos. Também, foram desconsideradas as espécies não identificadas, indeterminadas ou mortas.

As 11 listas de espécies foram convertidas em duas matrizes, sendo a primeira de presença/ausência, utilizada para a análise usual. e a segunda, baseada 
Quadro 1 - Informações sobre os 11 trabalhos realizados em Floresta Atlântica brasileira, códigos de identificação (C), localidade, localização geográfica, temperatura média (TM), critério de inclusão (CI), altitude média (AM), área total (AT), área amostral (AA), índice de diversidade de Shannon ( $\mathrm{H}^{\prime}$ ) e referência bibliográfica Table 1 - Information on the eleven works accomplished at Brazilian Atlantic Forest, identification codes $(C)$, place, geographical location, average temperature (TM), inclusion criterion (CI), average altitude (AM), total area (AT), sampling area $(A A)$, Shannon diversity index $\left(H^{\prime}\right)$ and references

\begin{tabular}{|c|c|c|c|c|c|c|c|c|c|c|}
\hline $\mathrm{C}$ & Formação Florestal & Localidade & Localização & $\begin{array}{l}\mathrm{TM} \\
\left({ }^{\circ} \mathrm{C}\right) \\
\end{array}$ & C. I. $(\mathrm{cm})$ & $\begin{array}{l}\mathrm{AM} \\
(\mathrm{m}) \\
\end{array}$ & AT (ha) & $\begin{array}{l}\text { AA } \\
\text { (ha) } \\
\end{array}$ & $\mathrm{H}^{\prime}$ & $\begin{array}{c}\text { Referência } \\
\text { Bibliográfica } \\
\end{array}$ \\
\hline M1 & $\begin{array}{c}\text { Floresta } \\
\text { Ombrófila } \\
\text { Densa }\end{array}$ & $\begin{array}{l}\text { Mata das Caldeiras } \\
\text { Catende, PE }\end{array}$ & $08^{\circ} 40^{\prime} \mathrm{S}$ e $35^{\circ} 35^{\prime} \mathrm{W}$ & 22 & $\mathrm{CAP} \geq 15$ & 169 & 38,56 & 1,00 & 3,83 & $\begin{array}{l}\text { Costa Junior } \\
\text { (2006) }\end{array}$ \\
\hline M2 & $\begin{array}{l}\text { Floresta } \\
\text { Ombrófila } \\
\text { Densa }\end{array}$ & $\begin{array}{l}\text { Matas do Curado } \\
\text { Recife, PE }\end{array}$ & $08^{\circ} 04^{\prime} \mathrm{S}$ e $34^{\circ} 55^{\prime} \mathrm{W}$ & 25 & $\mathrm{DAP} \geq 5$ & - & 100,86 & 1,00 & 3,66 & Espig (2003) \\
\hline M3 & $\begin{array}{l}\text { Floresta } \\
\text { Ombrófila } \\
\text { Densa }\end{array}$ & $\begin{array}{c}\text { Jardim Botânico do } \\
\text { Recife } \\
\text { Recife, PE }\end{array}$ & $08^{\circ} 04^{\prime} \mathrm{S}$ e $34^{\circ} 55^{\prime} \mathrm{W}$ & 25 & $\mathrm{CAP} \geq 15$ & 20 & 10,72 & 1,00 & 3,65 & $\begin{array}{l}\text { Sousa Junior } \\
\text { (2006) }\end{array}$ \\
\hline M4 & $\begin{array}{l}\text { Floresta } \\
\text { Ombrófila } \\
\text { Densa } \\
\text { Floresta }\end{array}$ & $\begin{array}{c}\text { RESEC Gurjaú } \\
\text { Cabo de Sto } \\
\text { Agostinho, PE } \\
\text { Mata do Sirigi }\end{array}$ & $08^{\circ} 10^{\prime} \mathrm{S}$ e $35^{\circ} 05^{\prime} \mathrm{W}$ & 24,7 & $\mathrm{CAP} \geq 15$ & 150 & 1077,1 & 1,00 & 3,91 & $\begin{array}{l}\text { Silva Junior } \\
\text { (2004) }\end{array}$ \\
\hline M5 & $\begin{array}{c}\text { Ombrófila } \\
\text { Montana } \\
\text { Floresta }\end{array}$ & $\begin{array}{l}\text { São Vicente Férrer, } \\
\text { PE }\end{array}$ & $07^{\circ} 38^{\prime} \mathrm{S}$ e $35^{\circ} 30^{\prime} \mathrm{W}$ & 24 & $\mathrm{DAP} \geq 5$ & 600 & 600 & 1,00 & - & Ferraz (2002) \\
\hline M6 & $\begin{array}{c}\text { Estacional } \\
\text { Semidecidual } \\
\text { Montana } \\
\text { Floresta }\end{array}$ & $\begin{array}{l}\text { Mata da Lagoa } \\
\text { Lavras, MG }\end{array}$ & $21^{\circ} 13^{\prime} \mathrm{S}$ e $44^{\circ} 58^{\prime} \mathrm{W}$ & 19,3 & $\mathrm{DAP} \geq 5$ & 884 & 4,0 & 1,16 & 3,89 & $\begin{array}{l}\text { Machado et. al. } \\
\text { (2004) }\end{array}$ \\
\hline M7 & $\begin{array}{c}\text { Estacional } \\
\text { Semidecidual } \\
\text { Montana }\end{array}$ & $\begin{array}{l}\text { Mata do Galego } \\
\text { Luminárias, MG }\end{array}$ & $21^{\circ} 29^{\prime} \mathrm{S}$ e $44^{\circ} 55^{\prime} \mathrm{W}$ & 19,6 & $\mathrm{CAP} \geq 15,5$ & 957 & 77,0 & 1,28 & 4,23 & $\begin{array}{l}\text { Rodrigues et. al. } \\
\text { (2003) }\end{array}$ \\
\hline M8 & $\begin{array}{c}\text { Floresta } \\
\text { Estacional } \\
\text { Semidecidual } \\
\text { Montana }\end{array}$ & $\begin{array}{l}\text { Fragmento } \\
\text { Florestal } \\
\text { Viçosa, MG }\end{array}$ & $20^{\circ} 45^{\prime} \mathrm{S}$ e $42^{\circ} 55^{\prime} \mathrm{W}$ & 18 & $\mathrm{CAP} \geq 15$ & - & 48 & 0,5 & 3,56 & Silva et. al. (2004) \\
\hline M9 & $\begin{array}{c}\text { Floresta Ombrófila } \\
\text { Densa }\end{array}$ & $\begin{array}{c}\text { Estação Ecológica } \\
\text { Estadual do Paraíso } \\
\text { Cachoeiras de } \\
\text { Macacus, RJ }\end{array}$ & $22^{\circ} 29^{\prime} \mathrm{S}$ e $42^{\circ} 55^{\prime} \mathrm{W}$ & 23 & $\mathrm{DAP} \geq 5$ & 200 & 4920 & - & 4,2 & $\begin{array}{l}\text { Kurtz e Araújo } \\
\text { (2000) }\end{array}$ \\
\hline M10 & $\begin{array}{c}\text { Floresta } \\
\text { Estacional } \\
\text { Semidecidual }\end{array}$ & $\begin{array}{l}\text { Fazenda Canchim } \\
\text { São Carlos, SP }\end{array}$ & $21^{\circ} 55^{\prime} \mathrm{S}$ e $47^{\circ} 52^{\prime} \mathrm{W}$ & 20 & $\mathrm{DAP} \geq 5$ & 850 & 112 & 0,02 & & $\begin{array}{c}\text { Silva e Soares } \\
\text { (2002) }\end{array}$ \\
\hline M11 & $\begin{array}{c}\text { Floresta Ombrófila } \\
\text { Densa }\end{array}$ & $\begin{array}{c}\text { Estação Florestal } \\
\text { Experimental } \\
\text { Nísia Floresta, RN }\end{array}$ & $06^{\circ} 55^{\prime} \mathrm{S}$ e $35^{\circ} 12^{\prime} \mathrm{W}$ & 24 & $\mathrm{DAP} \geq 5$ & 30 & 175 & 0,375 & 3,41 & $\begin{array}{l}\text { Oliveira et. al. } \\
\text { (2001) }\end{array}$ \\
\hline
\end{tabular}

na densidade e dominância absolutas das 237 espécies, utilizada para proposição de uma nova análise.

\subsection{Métodos Estatísticos}

Foram aplicadas duas metodologias de análise multivariada: 1) A técnica de Análise de Agrupamento; e 2) Análise de agrupamento precedida de análise de componentes principais, com o intuito de fazer uma comparação com os respectivos resultados.

$\mathrm{Na}$ primeira metodologia para a análise de agrupamento, a partir da matriz binária, representando a presença/ausência das espécies em cada área, utilizouse a matriz de similaridade obtida pelo cálculo coeficiente de comunidade de Jaccard. Na elaboração do dendrograma, utilizou-se o método de ligação simples ou método do vizinho mais próximo.

Na segunda metodologia a análise de componentes principais foi aplicada visando reduzir a dimensão do conjunto das variáveis originais. A partir dos escores dos componentes principais foi obtida a matriz de distância euclidiana entre as formações. Com base nessa matriz, aplicou-se o método de ligação simples para a formação dos grupos.

\subsubsection{Metodologia Usual}

\section{Análise de Agrupamento Simples}

Foi considerada a matriz de dados binária (237 espécies x 11 matas), em que na interseção das linhas 
e colunas se situou o valor de presença de cada atributo da comunidade arbórea, em que, por convenção, designou-se $1=$ espécie presente e $0=$ espécie ausente. Cada linha e coluna da matriz X descreveram-se, respectivamente, um vetor espécie $\left(X_{237}\right)$ e um vetor matas $\left(\mathrm{X}_{11}\right)$.

Para a comparação da florística entre os fragmentos realizou-se análise de agrupamento, por meio do método do vizinho mais próximo, bem como o cálculo do coeficiente de Jaccard (SOUZA et al., 1997) como medida de similaridade.

O coeficiente de comunidade ou similaridade de Jaccard, que relaciona o número de espécies comuns e o número de espécies encontradas em duas matas que se comparam, foi calculado por meio da fórmula:

$$
\mathrm{CCJ}_{i, j}=\frac{a}{a+b+c}
$$

em que: $\mathrm{CCJ}_{\mathrm{ij}}=$ coeficiente de comunidade de Jaccard para comparação das matas i e j $(\mathrm{i}, \mathrm{j}=1,2, \ldots, 11)$; $\mathrm{a}=$ número de espécies comuns as matas i e j $(\mathrm{i}, \mathrm{j}=1,2, \ldots, 11)$; $\mathrm{b}=$ número de espécies exclusivas da mata $\mathrm{i}$; e $\mathrm{c}=$ número de espécies exclusivas da mata j.

O coeficiente de comunidade de Jaccard apresenta valor 1 se as amostras são idênticas e valor 0 se são completamente distintas.

De posse dos dados desse coeficiente, foi construída a matriz de similaridade $\mathrm{S}$ de dimensão 11 $\mathrm{x} 11$.

Para a delimitação dos grupos, foi utilizado o método de ligação simples, um dos mais simples, de uso geral e de rápida aplicação.

De posse da matriz de similaridade de Jaccard $\left(\mathrm{S}_{\mathrm{ij}}\right)$, o método de ligação simples foi resolvido na seguinte sequiência de cálculos: 1) localizou-se o valor máximo de $S_{i j}<1$. As matas $M_{i}$ e $M_{j}$, correspondentes a esse valor, foram reunidas em um mesmo grupo, ficando (n-1) agrupamentos remanescentes; 2) com base na matriz de similaridade $\left(S_{1}\right)$, determinou-se a similaridade entre o novo agrupamento e as demais matas, por meio da relação: $S_{(i, j)} I=\operatorname{máx}\left(S_{i 1}, S_{i 2}\right), I=1,(n-2), I$ \# i \# j, e construiu-se nova matriz de similaridade $\left.\left(S_{2}\right) ; 3\right)$ localizou-se em $\mathrm{S}_{2}$ o maior valor de $\mathrm{Sij}<1$ e, em seguida, agruparam-se as matas que deram origem a essa nova similaridade, formando-se novo agrupamento. Nesse passo, têm-se (n-2) agrupamentos; e 4) compôs-se nova matriz de similaridade, com base na matriz $\mathrm{S}_{2}$. Para isso, calculou-se a similaridade entre o agrupamento formado na etapa anterior e os demais, considerando-se um elemento isolado de $\mathrm{M}$ como um agrupamento. Retornouse a seguir a etapa 3 . Os processos foram repetidos até que todas as 11 matas fossem alocadas a um só agrupamento.

\subsubsection{Metodologia Proposta}

Análise de Agrupamento precedida de Análise de Componentes Principais

Os dados originais foram padronizados visando minimizar os efeitos das diferentes escalas de mensuração. A padronização foi realizada utilizando-se a média $X_{i j}$ do j-ésima variável $(\mathrm{j}=1,2)$, avaliada na i-ésima espécie ( $i=1,2, \ldots ., 237)$, gerando a média reduzida de variância unitária (CRUZ e REGAZZI, 1997):

$$
Z_{i j}=\frac{X_{i j}}{S_{(? i)}}
$$

Os componentes principais são combinações lineares de variáveis, construídas de maneira a captar o máximo da variância, em que o primeiro componente explica a maior variação existente, o segundo componente explica a segunda maior variação e, assim, sucessivamente. A técnica consistirá na transformação de um conjunto de $\mathrm{n}$ variáveis padronizadas, $\mathrm{x}_{\mathrm{i} 1}, \mathrm{x}_{\mathrm{i} 2}$, ..., xin em um novo conjunto $\mathrm{y}_{\mathrm{i} 1}, \mathrm{y}_{\mathrm{i} 2}, \ldots$, yin, em que os yi's são funções lineares dos $\mathrm{x}_{\mathrm{i}^{\prime} \text { 's }}$ e independentes entre si. As seguintes propriedades foram verificadas:

a) $\mathrm{Se} \mathrm{Y}_{\mathrm{ij}}$ é um componente principal, então:

$$
\mathrm{Y}_{\mathrm{ij}}=\mathrm{a}_{1} \mathrm{x}_{\mathrm{i} 1}+\mathrm{a}_{2} \mathrm{x}_{\mathrm{i} 2}+\ldots+\mathrm{a}_{\mathrm{n}} \mathrm{x}_{\mathrm{in}}
$$

b) Se $Y_{i j}^{\prime}$ é outro componente principal então:

$\mathrm{Y}_{\mathrm{ij}}^{\prime}=\mathrm{b}_{1} \mathrm{x}_{\mathrm{i} 1}+\mathrm{b}_{2} \mathrm{x}_{\mathrm{i} 2}+\ldots+\mathrm{b}_{\mathrm{n}} \mathrm{x}_{\mathrm{in}}$

$\sum_{j} a_{j}^{2}=\sum_{j} b_{j}^{2}=1 ; \sum_{j} a_{j} b_{j}=0$, ou seja, os componentes são independentes.

c) Os componentes principais foram obtidos pela solução do sistema:det $\left(\mathrm{R}-?_{1} \mathrm{I}\right) \mathrm{a}=0$, em que: $\mathrm{R}=$ matriz de correlação entre as médias estimadas, $\lambda_{1}=$ raízes características ou (autovalores) de R, I = matriz-identidade de dimensão pxp; e a = vetor característico (ou autovetor) 
associado aos autovalores (HOFFMANN, 1999). Dessa forma, os autovalores de $\mathrm{R}$ correspondem às variâncias de cada componente e os autovetores normalizados, aos coeficientes de ponderação dos caracteres padronizados.

A importância relativa de um componente, que é avaliada pela porcentagem da variação total que esse explica, foi calculada por:

$$
\text { Importância de } \mathrm{Y}_{\mathrm{j}}=\frac{?_{\mathrm{j}}}{\operatorname{traço}(\mathrm{R})}
$$

Depois da determinação dos componentes principais, foram estimados os respectivos escores associados a cada mata estudada. Com base nesses escores, foi obtida a distância euclidiana média, como medida representativa da divergência entre aquelas matas.

A análise de agrupamento foi aplicada, utilizandose como medida de dissimilaridade a distância euclidiana.

A distância euclidiana foi estimada pela seguinte expressão:

$d_{i i^{\prime}}=\left[\sum_{j}\left(c p_{i j}-c p_{i^{\prime} j}\right)^{2}\right]^{1 / 2}$

em que $\mathrm{d}_{\mathrm{ii}^{\prime}}=\mathrm{a}$ distância euclidiana entre as matas i e i'; $\mathrm{cp}_{\mathrm{ij}}-\mathrm{cp}_{\mathrm{i}^{\prime} \mathrm{j}}=$ diferença entre escores dos componentes principais das matas i e $i^{\prime}$, para um componente $\mathrm{j}$.

De posse da matriz de distância euclidiana $\left(\mathrm{D}_{\mathrm{ij}}\right)$, o método de ligação simples foi resolvido na seguinte seqüência de cálculos:

1) Localizou-se o valor mínimo de $D_{i j}>0$. As matas $M_{i}$ e $M_{j}$, correspondentes a esse valor, foram reunidas em um mesmo grupo, ficando (n-1) agrupamentos remanescentes; 2) Com base na matriz de distância $\left(D_{1}\right)$, determinou-se a similaridade entre o novo agrupamento e as demais matas, por meio da relação: $\mathrm{D}_{(\mathrm{i}, \mathrm{j})} \mathrm{I}=$ mínimo $\left(\mathrm{D}_{\mathrm{i} 1}, \mathrm{D}_{\mathrm{i} 2}\right), \mathrm{I}=1$, ( $\left.\mathrm{n}-2\right), \mathrm{I} \# \mathrm{i} \# \mathrm{j}$, e construiuse nova matriz de similaridade $\left(\mathrm{D}_{2}\right) ; 3$ ) Localizou-se em $\mathrm{D}_{2}, \mathrm{o}$ menor valor de $\mathrm{D}_{\mathrm{ij}}>0 \mathrm{e}$, em seguida, agruparamse as matas que deram origem a essa nova similaridade, formando-se novo agrupamento. Nesse passo, têmse (n-2) agrupamentos; e 4) Compôs-se nova matriz de similaridade, baseando-se na matriz $\mathrm{D}_{2}$. Para isso, calculou-se a similaridade entre o agrupamento formado na etapa anterior e os demais, considerando-se um elemento isolado de $\mathrm{M}$ como um agrupamento. Retornouse a seguir à etapa 3). Os processos foram repetidos até que todas as 11 matas fossem alocadas a um só agrupamento.

As análises estatísticas dos dados foram processadas por meio dos softwares Microsoft Excel 2003 para Windows XP, pacote estatístico SAS 8.0 e do MINITAB 14.0.

\section{RESULTADOS E DISCUSSÃO}

\subsection{Metodologia Usual}

Quanto à similaridade florística dos fragmentos, observou-se o coeficiente de Jaccard variando de 0,00 a 0,38, indicando maior semelhança entre a Mata das Caldeiras e do Jardim Botânico (Quadro 2). Observase, nesse quadro, que há uma tendência de menor similaridade entre as Matas do Nordeste e as do Sudeste (Figura 1), com a definição de um grupo formado pelas Matas 1, 2, 3, 4 e 5 (matas de Pernambuco), outro grupo pelas Matas 6 e 7 (Minas Gerais). Tal resultado corrobora as afirmativas de Scudeller (2002) de que há maior similaridade entre matas do Sul-Sudeste do que Nordeste-Sudeste e de Siqueira (1994), em que há existência de dois blocos florísticos bem individualizados para a Floresta Atlântica no Brasil, um formado pela Região Nordeste e outro pela Região Sudeste/Sul.

Para a maioria das comparações entre as áreas, o coeficiente de Similaridade de Jaccard obtido neste estudo foi baixo, pois, segundo Mueller-Dombois e Ellenberg (1974), esse coeficiente raramente atinge valores acima de $60 \%$ e deve ser superior a $25 \%$, para que duas formações florestais sejam consideradas similares. De acordo com Oliveira e Rotta (1982), fatores que promovem resultados de índices muito baixos entre algumas áreas podem ser: diferenças de solo, exploração concentrada em parte da área ou até diferença de sítio. O segundo fator parece ser o que mais contribuiu para esse valor baixo de similaridade florística. Para Bertoni e Martins (1987), as variações florísticas estariam relacionadas à heterogeneidade ambiental e aos diferentes graus de perturbação a que essas florestas são submetidas. Isso indica que essas variações podem ser conseqüências da heterogeneidade ambiental ao longo da paisagem e não simplesmente um efeito da fragmentação (SANTOS, 2003).

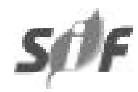

R. Árvore, Viçosa-MG, v.32, n.03, p.511-521, 2008 
Quadro 2 - Matriz de similaridade obtida por meio do coeficiente de Jaccard (S) das Matas das Caldeiras (M1), do Curado (M2), do Jardim Botânico (M3), da Reserva de Gurjaú (M4), do Sirigi (M5), da Lagoa (M6), do Galego (M7), de Viçosa (M8), da Estação do Paraíso (M9), da Fazenda Canchim (M10) e da Estação de Nísia Floresta (M11)

Table 2 - Similarity matrix using the Jaccard's coefficient (S) for Mata das Caldeiras (M1), Curado(M2), Botanical Garden (M3), Gurjaú Reserve (M4), Sirigi (M5), Lagoa (M6), Galego (M7), Viçosa (M8), Paraíso Station (M9), Fazenda Canchim (M10) and Nísia Forest Station (M11)

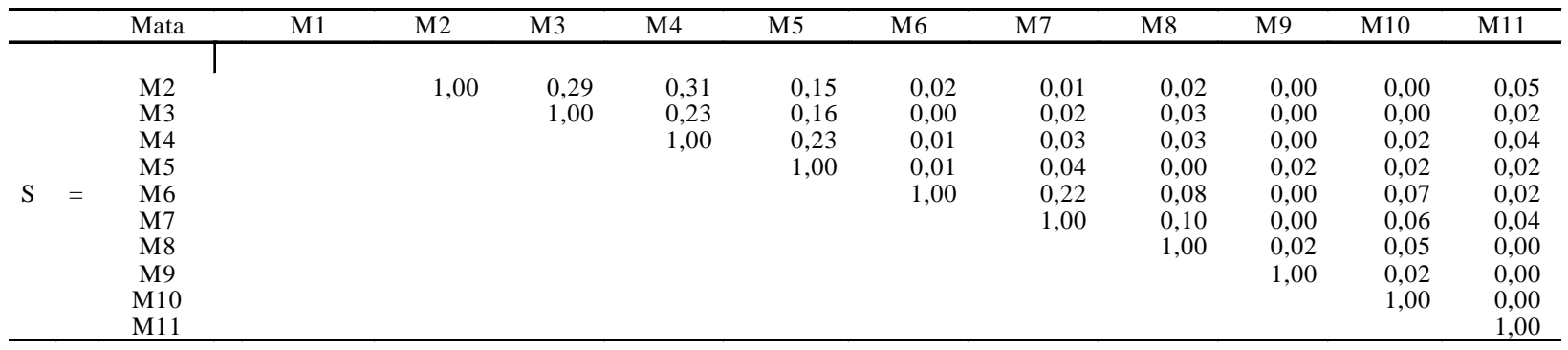

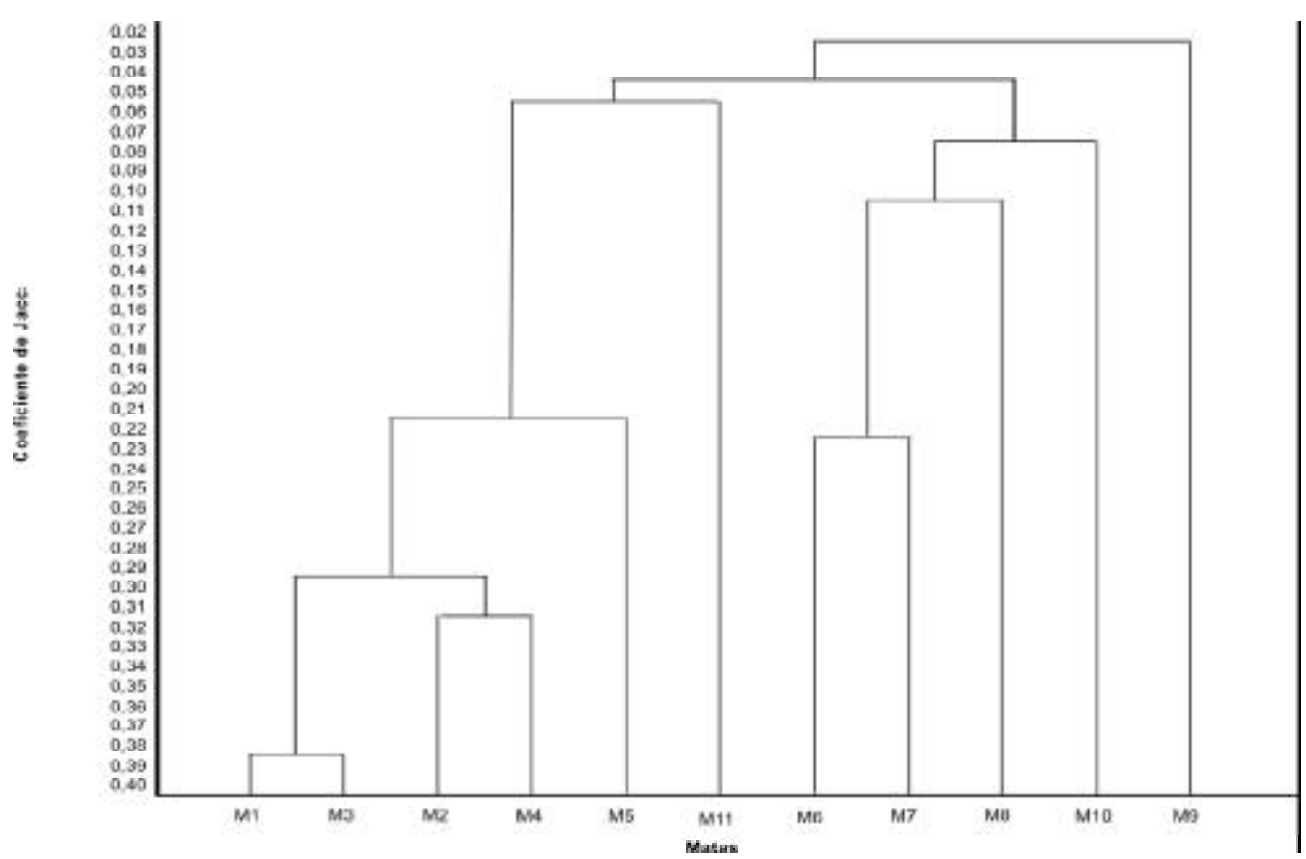

Figura 1 - Dendrograma representando as seqüências de agrupamentos das 11 matas, obtidas pelo método de ligação simples, com base no coeficiente de Jaccard (Matas das Caldeiras (M1), do Curado (M2), do Jardim Botânico (M3), da Reserva de Gurjaú (M4), do Sirigi (M5), da Lagoa (M6), do Galego (M7), de Viçosa (M8), da Estação do Paraíso (M9), da Fazenda Canchim (M10) e da Estação de Nísia Floresta (M11)).

Figure 1 -Dendrogram representing the sequences of groupings of the 11 forests, obtained by the complete linkage method, based on Jaccard's similarity coefficient for Mata das Caldeiras (M1), Curado(M2), Botanical Garden (M3), Gurjaú Reserve (M4), Sirigi (M5), Lagoa (M6), Galego (M7), Viçosa (M8), Paraíso Station (M9), Fazenda Canchim (M10) and Nísia Forest Station (M11).

Os resultados, de certa forma, confirmam as idéias de Condit (1996) de que seria simplificar demais achar que a heterogeneidade entre florestas possa ser devida somente a fatores ambientais e que os diferentes efeitos da fragmentação sobre a composição de florestas dependem da escala temporal adotada, pois a comunidade pode nunca chegar a um equilíbrio na composição de espécies em qualquer escala de tempo ou espaço. 
Cada fragmento exibe uma composição de espécies que parece resultante de uma série de fatores que variaram diferentemente ao longo do espaço e do tempo. Talvez por isso seja tão difícil estabelecer dessas matas. Essa dificuldade, entretanto, indica que cada fragmento apresenta um conjunto de características próprias, o que ressalta sua importância em termos de conservação.

\subsection{Metodologia Proposta}

A redução da dimensionalidade proporcionada pelas técnicas de componentes principais se encontra ilustrada no Quadro 3. Pode-se observar que foi possível a redução da 474a para a décima dimensão, com a acumulação de $100,00 \%$ da variação total nos 10 primeiros componentes.

Com base nos resultados, justifica-se a utilização das análises de componentes principais, por proporcionarem uma simplificação estrutural dos dados originais. Os 10 primeiros componentes principais explicaram 100\% da variância, indicando que os 462 componentes principais restantes são combinações lineares dos 10 primeiros. Portanto, usar os 10 primeiros componentes é tão eficiente quanto o uso das 472 variáveis iniciais, no que se refere à explicação da variância.

Utilizando-se dos escores referentes aos 10 primeiros componentes, foi realizada a análise de agrupamento.

Quanto à similaridade de estrutura dos fragmentos, observou-se distância euclidiana variando de 23,32 a 39,00 (Quadro 4), o que indica maior semelhança entre as Matas do Curado e do Jardim Botânico e maior diferença entre as Matas do Galego e de Viçosa.

A semelhança entre as Matas do Curado e do Jardim Botânico era esperada devido à proximidade geográfica. Jacquemyn et al. (2001) também observaram que a similaridade florística diminuía com o aumento da distância entre as áreas, em concordância com as idéias de Condit (1998) citado por Cook (1998), segundo o qual a proximidade geográfica seria o único fator confiável para se prever a similaridade entre áreas.

Na Figura 2, observa-se a definição de um grupo formado pelas matas 1, 2, 3 e 4 (matas de Pernambuco) e outros sete grupos formados por matas isoladas.

\subsection{Metodologia Usual $x$ Metodologia Proposta}

Na metodologia usual, com base no coeficiente de Jaccard (Quadro 2) foi verificada maior semelhança entre a Mata das Caldeiras e do Jardim Botânico. Já com base na distância euclidiana (Quadro 3), a maior semelhança foi entre as Matas do Curado e as do Jardim Botânico. O segundo resultado é o mais esperado tendo em vista a proximidade das matas, o que não foi detectado pela primeira metodologia. O que ressalta a potencialidade do uso da metodologia proposta.

De maneira geral, as visualizações dos respectivos dendrogramas (Figuras 2 e 3 ) demonstram as variações realizadas pelas análises de agrupamento através dos seus índices de similaridade, e medidas de distância para os cálculos das comparações florísticas e comparação da estrutura arbórea das regiões de mata atlântica em estudo.

Quadro 3 - Estimativas das variâncias (autovalores, $\lambda \mathrm{j}$ ) associadas aos componentes principais e suas importâncias relativas e acumuladas

Table 3 - Variance estimates (eigenvalues, $l j$ ) associated with principal components and their relative and accumulated importances

\begin{tabular}{cccc}
\hline Componentes Principais & Autovalor $\left(\lambda_{\mathrm{j}}\right)$ & $\begin{array}{c}\text { Proporção da Variância } \\
(\%)\end{array}$ & $\begin{array}{c}\text { Proporção Acumulada da Variância }(\%) \\
1\end{array}$ \\
2 & 97,33 & 20,62 & 20,62 \\
3 & 62,14 & 13,16 & 33,79 \\
4 & 53,37 & 11,31 & 45,09 \\
5 & 47,28 & 10,02 & 55,11 \\
6 & 44,28 & 9,38 & 64,49 \\
7 & 39,91 & 8,46 & 72,95 \\
8 & 37,86 & 8,02 & 80,97 \\
9 & 34,37 & 7,28 & 88,25 \\
10 & 31,27 & 6,62 & 94,87 \\
11 a 472 & 24,19 & 5,13 & 100,00 \\
\end{tabular}


Quadro 4 - Matriz de distância euclidiana obtida com base nos escores dos componentes principais das Matas das Caldeiras (M1), do Curado (M2), do Jardim Botânico (M3), da Reserva de Gurjaú (M4), do Sirigi (M5), da Lagoa (M6), do Galego (M7), de Viçosa (M8), da Estação do Paraíso (M9), da Fazenda Canchim (M10) e da Estação de Nísia Floresta (M11)

Table 4 - Matrix of Euclidean distances based on scores of principal components for Mata das Caldeiras (M1), Curado(M2), Botanical Garden (M3), Gurjaú Reserve (M4), Sirigi (M5), Lagoa (M6), Galego (M7), Viçosa (M8), Paraíso Station (M9), Fazenda Canchim (M10) and Nísia Forest Station (M11)

\begin{tabular}{|c|c|c|c|c|c|c|c|c|c|c|c|c|c|}
\hline & & Mata & M1 & M2 & M3 & M4 & M5 & M6 & M7 & M8 & M9 & M10 & M11 \\
\hline \multirow{11}{*}{$S$} & & M1 & 0,00 & 24,04 & 25,14 & 27,74 & 28,24 & 31,11 & 37,96 & 32,17 & 27,95 & 29,65 & 27,23 \\
\hline & & M2 & & 0,00 & 23,32 & 24,15 & 27,07 & 28,93 & 36,61 & 30,39 & 25,84 & 27,67 & 25,45 \\
\hline & & M3 & & & 0,00 & 27,56 & 28,52 & 30,45 & 37,55 & 31,29 & 27,18 & 28,93 & 26,89 \\
\hline & & M4 & & & & 0,00 & 28,22 & 31,76 & 38,52 & 32,81 & 28,92 & 30,53 & 28,29 \\
\hline & & M5 & & & & & 0,00 & 31,87 & 38,53 & 33,09 & 28,38 & 30,28 & 28,58 \\
\hline & $=$ & M6 & & & & & & 0,00 & 36,80 & 33,04 & 29,84 & 30,81 & 29,48 \\
\hline & & M7 & & & & & & & 0,00 & 39,00 & 37,11 & 37,69 & 36,52 \\
\hline & & M8 & & & & & & & & 0,00 & 30,84 & 32,08 & 30,93 \\
\hline & & M9 & & & & & & & & & 0,00 & 27,86 & 26,29 \\
\hline & & M10 & & & & & & & & & & 0,00 & 28,09 \\
\hline & & M11 & & & & & & & & & & & 0,00 \\
\hline
\end{tabular}

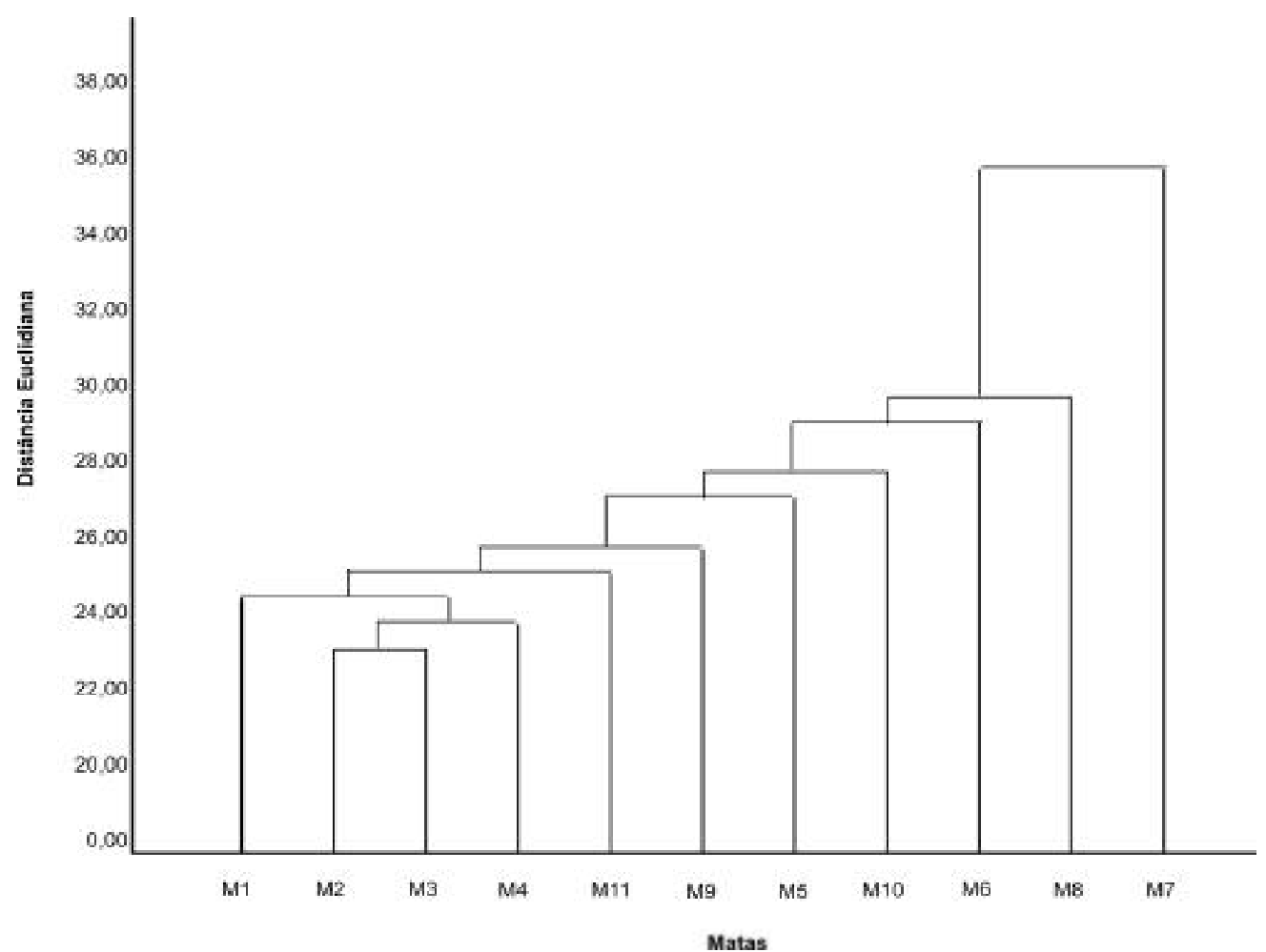

Figura 2 - Dendrograma representando as seqüências de agrupamentos das 11 matas, obtidas pelo método de ligação simples, com base na distância euclidiana (Matas das Caldeiras (M1), do Curado (M2), do Jardim Botânico (M3), da Reserva de Gurjaú (M4), do Sirigi (M5), da Lagoa (M6), do Galego (M7), de Viçosa (M8), da Estação do Paraíso (M9), da Fazenda Canchim (M10) e da Estação de Nísia Floresta (M11)).

Figure 2-Dendrogram representing the sequences of groupings of the 11 forests, obtained by the complete linkage method, based on the Euclidean distance for Mata das Caldeiras (M1), Curado(M2), Botanical Garden (M3), Gurjaú Reserve (M4), Sirigi (M5), Lagoa (M6), Galego (M7), Viçosa (M8), Paraíso Station (M9), Fazenda Canchim (M10) and Nísia Forest Station (M11).

R. Árvore, Viçosa-MG, v.32, n.03, p.511-521, 2008 
$\mathrm{Na}$ análise de similaridade florística (Figura 2), a maioria das matas da região Nordeste se concentram em um grupo bem distinto, ocorrendo o mesmo com os da Região Sudeste. O que não ocorre quando aplicada à medida de distância (Figura 3), na qual os grupos da Região Nordeste ainda prevalecem, porém com certas variações para as formações dos grupos, enquanto as matas da Região Sudeste sofreram alterações, inclusive incluindo mata da Região Nordeste, o que não ocorreu no índice de similaridade.

Vale salientar que, para os coeficientes de similaridade, um valor alto indica proximidade ou alta similaridade, enquanto para as medidas de distância um valor baixo é que indica a proximidade ou alta similaridade.

Com isso, fica evidente que, para a comparação florística em um conjunto de dados, as variáveis quantitativas são significativamente importantes na identificação dos grupos que mais se aproximam, que no referido trabalho se trata da densidade absoluta (DA) e dominância absoluta (DoA) das espécies em cada região de Mata Atlântica. O mesmo não ocorre na matriz binária, já que se trata apenas de identificar se determinada espécie pertence ou não a determinada região, independentemente de sua abundância.

\section{CONCLUSÃO}

- A metodologia proposta tem potencial para utilização no estudo de similaridade de fragmentos florestais.

- Variáveis quantitativas devem ser consideradas no estudo de similaridade entre fragmentos florestais.

- Recomenda-se utilizar maior número de variáveis para exprimir a heterogeneidade ambiental entre fragmentos.

\section{REFERÊNCIAS}

BERTONI, J.E.A., MARTINS, F.R. Composição florística de uma floresta ripária na Reserva Estadual de Porto Ferreira,SP. Acta Botânica Brasílica, v.1, p.17-26, 1987.

CONDIT, R. Defining and mapping vegetation types in mega-diverse tropical forests. Trends in Ecology and Evolution, v.11, n.1, p.4-5, 1996.
COOK, S. A diversity of approaches to the study of species richness. Trends in Ecology and Evolution, v.13, n.9, p.340-341, 1998.

COSTA JUNIOR, R. F. Caracterização estrutural de um remanescente de mata atlântica do município de Catende - PE. Recife, 2006. 52f. Dissertação (Mestrado em Ciências Florestais) - Universidade Federal Rural de Pernambuco.

CRUZ, C. D.; REGAZZI, A. J.Métodos biométricos aplicados ao melhoramento genético. Viçosa: UFV, 1997, 390p.

DISLICH, R., CERSÓSIMO, L., MANTOVANI, W. Análise da estrutura de fragmentos florestais no planalto paulistano - SP. Revista Brasileira de Botânica, v.24, n.3, p.321-332, 2001.

ESPIG, S. A. Distribuição de nutrientes em fragmento de Mata Atlântica em Pernambuco Recife, 2003. 52f. Dissertação (Mestrado em Ciências do Solo) - Universidade Federal Rural de Pernambuco.

FERRAZ, E. M. N. Estudo florístico e fitossociológico de um remanescente de floresta ombrófila densa em Pernambuco, Nordeste do Brasil Recife, 2002. 146f. Tese (Doutorado em Botânica) Universidade Federal Rural de Pernambuco.

FERREIRA, L.V., PRANCE, G.T. 1998. Species richness and floristic composition in four hectares in the Jaú National Park in upland forests in Central Amazonia. Biodiversity and Conservation, v.7, n.10, p.1349-1364, 1998.

HOFFMANN, R. Componentes principais e análise fatorial. 4. ed. Piracicaba: ESALQ/ USP, 1999. 40P. (Série didática, $\mathrm{n}^{\circ}$ 90).

JACQUEMYN, H., BUTAYE, J., DUMORTIER, M., HERMY, M. e LUST, N. Effects of age and distance on the composition of mixed deciduous forest fragments in an agricultural landscape. Journal of Vegetation Science, v.12, n.5, p.635-642, 2001.

JOLY, C.A., LEITÃO FILHO, H.F., SILVA, S.M. O patrimônio florístico. In: Mata Atlântica. CÂMARA, G. (Ed.). São Paulo: Index e S.O.S Mata Atlântica, 1991. p.9-128. 
KURTZ, B. C.; ARAÚJO, D. S. D. Composição florística e estrutura do componente arbóreo de um trecho de Mata Atlântica na Estação Ecológica Estadual do Paraíso, Cachoeiras de Macacu, Rio de Janeiro, Brasil. Revista Rodriguésia, v.51, n.78/115, p.69-112, 2000.

MACHADO, E. L. M.; FILHO, A. T. O. Análise comparativa da estrutura e flora do compartimento arbóreo-arbustivo de um remanescente florestal na fazenda Beira Lago, Lavras, MG. Revista Árvore, v.28, n.4, p.499-516, 2004.

MUELLER-DUMBOIS, D., ELLENBERG, H.Aims and methods vegetation ecology. New York: John Wiley e Sons, 1974. 547p.

OLIVEIRA FILHO, A.T., FONTES, M.A.L. Patterns of floristic differentiation among Atlantic Forest in south-eastern Brazil, end the influence of climate. Biotropica, v.32, n.5 (suppl.), p.1-16, 2000.

OLIVEIRA FILHO, A.T., RATTER, J.A. A study of the origin of Central Brazilian forests by the analysis of plant species distribution patterns. Edinburg Journal of Botany, v.52, n.2, p.141-194, 1995.

OLIVEIRA, Y. M. M.; ROTTA, E. Levantamento da estrutura horizontal de uma mata de Araucária do primeiro planalto paranaense.

Boletim de Pesquisa Florestal, Curitiba, v.4, p.1-46, 1982.

OLIVEIRA, Z. L.; SANTOS JUNIOR, R. C. B., FELICIANO, A.L.P., MARANGON, L.C., CARVALHO, A.J.E. de. Levantamento florístico e fitossociológico de um trecho de Mata Atlântica na Estação Florestal Experimental de Nísia Floresta - RN. Revista Brasil Florestal, v.20, n.71, p.22-29, 2001.

PEIXOTO, G.L., MARTINS, S.V., SILVA, A.F. da, SILVA, E. Composição florística do componente arbóreo de um trecho de Floresta Atlântica na Área de Proteção Ambiental da Serra da Capoeira Grande, Rio de Janeiro, RJ, Brasil. Acta

Botânica Brasílica, v.18, n.1, p.151-160, 2004.

RODRIGUES, L. A.; CARVALHO, D. A. Florística e estrutura da comunidade arbórea de um fragmento florestal em Luminárias, MG. Acta Botânica Brasílica, v.17, n.1, p.71-87, 2003.
SANTOS, K. Caracterização florística e estrutural de onze fragmentos de Mata Estacional Semidecidual da área de proteção ambiental do município de Campinas - SP. Campinas: Unicamp, 2003. 225f. Tese (Doutorado em Biologia Vegetal) Universidade Estadual de Campinas.

SCIAMARELLI, A. Estudo florístico e fitossociologia da "Mata de Dourados", fazenda Paradouro, Dourados, Mato Grosso do Sul, Brasil. Campinas: Unicamp, 2005. 120f. Tese (Doutorado em Biologia Vegetal) - Universidade Estadual de Campinas.

SCUDELLER, V.V. Análise fitogeográfica da mata atlântica - Brasil. Campinas:

Unicamp, 2002. 204f. Tese (Doutorado em Biologia Vegetal) - Universidade Estadual de Campinas .

SCUDELLER, V.V., MARTINS, F.R., SHEPHERD, G.J. Distribution and abundance of arboreal species in the Atlantic Ombrophilous Dense Forest in southeastern Brasil. Plant Ecology, v.152, n.2, p.185-199, 2001.

\section{SILVA JÚNIOR, J. F. Estudo}

fitossociológico em um remanescente de floresta atlântica visando dinâmica de espécies florestais arbóreas no município do Cabo de Santo Agostinho, PE. Recife, 2004. 82f. Dissertação (Mestrado em Ciências Florestais) Universidade Federal Rural de Pernambuco.

SILVA, N. R. S.; MARTINS, S. V.; MEIRA NETO, J. A. A.; et. al. Composição florística e estrutura de uma floresta estacional semidecidual montana em Viçosa, MG. Revista Árvore, v.28, n.3, p.397-405, 2004.

SILVA, A.F., SHEPHERD, G.J. Comparações florísticas entre algumas matas brasileiras utilizando análise de agrupamento. Revista Brasileira de Botânica, v.9, n.1, p.81-86, 1986.

SILVA, L. A.; SOARES, J. J. Estudo florístico e fitossocilógico em um fragmento de floresta estacional semidecídua, no município de São Carlos - SP. Acta Botânica Brasílica, v.16, n.2, p.205-216, 2002. 
SILVA, N. R. S.; MARTINS, S. V.; MEIRA NETO, J. A. A.; et. al. Composição florística e estrutura de uma floresta estacional semidecidual montana em Viçosa, MG. Revista Árvore, v.28, n.3, p.397-405, 2004.

SIQUEIRA, M.F. Análise florística e ordenação de espécies arbóreas da Mata Atlântica através de dados binários. Campinas; Unicamp, 1994. 142f. Dissertação (Mestrado em Ecologia) - Universidade Estadual de Campinas.

SOUSA JUNIOR, P. R. C. Estrutura da comunidade arbórea e da regeneração natural em um fragmento de floresta urbana, Recife - PE. Recife, 2006. $91 \mathrm{f}$. Dissertação (Mestrado em Ciências Florestais) Universidade Federal Rural de Pernambuco.
SOUZA, A. L.; FERREIRA, R. L. C.; XAVIER, A. Análise de Agrupamento Aplicada à Ciência Florestal. Viçosa: SIF, 1997. 109 f. (Documento SIF, 16).

VAN DEN BERG, E. e OLIVEIRA-FILHO, A. T. Composição florística e estrutura fitossociológica de uma floresta ripária em Itutinga, MG, e comparação com outras áreas. Revista Brasileira de Botânica, v.23, n.3, p.231-253, 2000.

Van TONGEREN, O.F.G. Cluster analysis. In: R.H.G. Jongman, C.J.F. ter Braak, O.F.R. van Tongeren (eds.) Data analysis in community and landscape Ecology. Pudoc, Wageningen, 1987. p.174-212. 